increase the quality and quality of clinical and translational research in Rhode Island, and to apply these findings to address barriers and strengthen research capabilities across our partner institutions. METHODS/STUDY POPULATION: We utilized a Group Concept Mapping approach, involving university and Institution-based researchers and administrators. The process was conducted using the web-based concept mapping application CS Global Max (Concept Systems, Inc). Respondents were asked to provide their best ideas for promoting clinical and translational research in RI. These ideas were then organized by our project team into a set of unique items for consideration by attendees of an Advance-CTR retreat. Participants were tasked with sorting these ideas by theme (cluster), and were also asked to rate each idea according its importance and feasibility. Using the online software, these clusters and ratings were analyzed to identify key themes and to explore differences among subgroups. RESULTS/ANTICIPATED RESULTS: The Group Concept Mapping exercise yielded 150 statements that were edited down to 78 unique ideas, and clustered into nine themes (e.g., institutional collaboration, training). Fifty-seven retreat participants completed the sorting and rating tasks of the concept mapping exercise. Overall, ideas rated as highly important and highly feasible included "providing seed grants to encourage new collaborations across basic science," and "connecting researchers with common interests." Top rated items varied across institutions and according to respondent demographics, allowing us to consider the unique issues relevant to particular groups. Relative rankings of clusters across groups revealed notable differences, such as higher importance placed on community engagement among administrators as compared with researchers, and differences in needs for internal support for research between universities. DISCUSSION/SIGNIFICANCE OF IMPACT: Group Concept Mapping was an effective and insightful participatory approach to engage our program's stakeholders in developing ideas and identifying challenges to enhancing clinical and translational research in Rhode Island. Our results have implications for project decision-making and initiatives to facilitate translational research in RI. Thus, results have been presented to the AdvanceCTR community via webinar, as well as Advance-CTR project leadership and advisory committees.

3209

\author{
Hepatitis C Virus Linked To Increased Mortality in \\ Inmates Who Are Hospitalized \\ Alysse G Wurcel ${ }^{1}$, Deirdre Burke ${ }^{2}$, Karen Freund ${ }^{1}$, Curt Beckwith ${ }^{3}$ \\ and John Wong ${ }^{1}$ \\ ${ }^{1}$ Tufts University; ${ }^{2}$ Tufts Medical Center and ${ }^{3}$ Brown University
}

OBJECTIVES/SPECIFIC AIMS: Hepatitis C virus (HCV) has a high prevalence among individuals in jail and prisons. Access to $\mathrm{HCV}$ treatment has been restricted in jails and prisons. We hypothesized that HCV infection in inmates would be associated with increased mortality in people who were hospitalized while incarcerated. METHODS/STUDY POPULATION: We created and then linked a database of people who were incarcerated and admitted at Lemuel Shattuck Hospital $(2004,2008,2011)$ to the Massachusetts Vital Statistic Registry (updated through end of 2015). Death was classified using the Automatic Classification of Medical Entry Death Code. The primary outcome of interest was mortality within 1 year of hospitalization, and the secondary outcome was mortality at any time. The primary indicator of interest was HCV, defined as the presence of the ICD-9 code for $\mathrm{HCV}$ on discharge. Covariates included in univariate and multivariate modeling included age, year of admission, and race/ethnicity classified as: White, Black, Hispanic or Other (i.e., Asian, Native American, Multi-Racial, or No answer). RESULTS/ANTICIPATED RESULTS: Of the 1,541 hospital admissions, $21 \%$ had HCV, and $57 \%$ were white, $22 \%$ black, $8 \%$ Hispanic and $12 \%$ other. Of the 273 total deaths (18\% of cohort), 82 deaths occurred within 1 year of hospitalization (5.3\% of the entire cohort, $30 \%$ of all deaths). The primary cause of death was vascular (21\%), followed by chronic liver disease (18\%), cancer (17\%), overdose/suicide/trauma (19\%), pulmonary (7\%) and infection (6\%). People with HCV were more likely to die of chronic liver disease (40\% vs $7 \%$, $\mathrm{p}<0.001)$. In the multivariable adjusted model, people with $\mathrm{HCV}$ were more likely to die within 1 year of hospitalization (HR 1.59, $95 \%$ CI 1.02, 2.49) and more likely to die at any time (HR 1.38, $95 \%$ CI 1.06, 1.79). Age, race and gender were not associated with risk of death. Compared to 2004, people admitted in 2008 (HR 2.05, 95\% CI, 1.50-2.80) and 2011 (HR 4.02, 95\% CI 2.77, 5.83) were more likely to die within 1 year. DISCUSSION/SIGNIFICANCE OF IMPACT: Despite advances in HCV treatment in the community, HCV in inmates is associated with increased mortality.

3294

\section{HIGH INTENSITY BINGE DRINKING AND STIMULATING EFFECTS IN HUMAN LABORATORY STUDIES OF ALCOHOL SELF-ADMINISTRATION}

Julia Swan ${ }^{1}$, Joshua L.Gowin, Bethany L. Stangl and Vijay

A. Ramchandani

${ }^{1}$ National Institutes of Health

OBJECTIVES/SPECIFIC AIMS: Alcohol use disorder (AUD) has previously been studied using Timeline Followback (TLFB) interview measures and administration of alcohol within laboratory sessions. However, most of those studies supplied alcohol orally and analyzed drinking across a range of drinking intensity and frequency measures. High intensity binge drinking, i.e., drinking alcohol at multiple levels of the binge threshold ( $5+$ drinks for males, $4+$ drinks for females) has been identified as a significant risk factor for developing AUD. In the present study, we examined the relationship between high intensity binge drinking with the behavioral and subjective response to intravenous alcohol in a lab study. METHODS/ STUDY POPULATION: Two hundred participants completed a 90-Day TLFB interview, wherein the maximum number of drinks in a day established the participant's binge level status as a Non-Binger $(\mathrm{N}=37)$, Binge Level $1(\mathrm{~N}=96)$, Binge Level $2(\mathrm{~N}=44)$, or Binge Level $3(\mathrm{~N}=22)$. Binge Level 1 corresponds with at least one binge (4-7 drinks for women, 5-9 drinks for men); Binge Level 2 requires at least twice the binge level (8-11 drinks for women, 10-14 drinks for men); and Level 3 necessitates a participant to drink at least three times the binge level (12+ drinks for women, $15+$ drinks for men) on one day. Non-Bingers had no binge level drinking in the 90-day interview. Participants also underwent a 150-minute intravenousalcohol self-infusion, where participants would press a button to receive an infusion of an ethanol solution. During this, participants also completed subjective questionnaires including the Alcohol Urge Questionnaire (AUQ), Biphasic Alcohol Effects Scale (BAES), and Drug Effects Questionnaire (DEQ). Kruskal-Wallis and chisquare tests were used to examine the effect of group on alcohol infusion and subjective response measures. RESULTS/ANTICIPATED RESULTS: A chi-square test for association showed significant statistical differences by groups in reaching binge level status $(0.08 \%$ breath alcohol content) during the alcohol infusion session in the lab, X2 $(3)=23.321, \mathrm{p}<0.001$. However, mean difference 